Portland State University

PDXScholar

Electrical and Computer Engineering Faculty

Publications and Presentations

$4-1-1981$

\title{
Laser Broadening Ratio from the Multimode Oscillation Threshold
}

Lee W. Casperson

Portland State University

Follow this and additional works at: https://pdxscholar.library.pdx.edu/ece_fac

Part of the Electrical and Computer Engineering Commons

Let us know how access to this document benefits you.

Citation Details

Casperson, L. W. (1981). Laser broadening ratio from the multimode oscillation threshold. Journal of Applied Physics, 52 (11), 6981-6983.

This Article is brought to you for free and open access. It has been accepted for inclusion in Electrical and Computer Engineering Faculty Publications and Presentations by an authorized administrator of PDXScholar. Please contact us if we can make this document more accessible: pdxscholar@pdx.edu. 


\title{
Laser broadening ratio from the multimode oscillation threshold
}

\author{
Lee W. Casperson
}

School of Engineering and Applied Science, University of California, Los Angeles, California 90024

(Received 20 March 1981; accepted for publication 16 April 1981)

Lasers with mixed line broadening typically oscillate in a single longitudinal mode for excitation levels only slightly above threshold. At high pump levels, less desirable multimode oscillation may sometimes occur. A procedure is described for using the multimode threshold as a sensitive measure of the broadening ratio, and GaAs is mentioned as an example.

PACS numbers: 42.55.Bi, 42.55.Px, 42.60.By, 42.60.Da

For many applications of laser oscillators, one requires that the optical signal be highly stable in amplitude and frequency. As a first step, it is important to ensure that the laser is oscillating in a single cavity mode. In some lasers, single mode oscillation occurs spontaneously, and in others, it can be obtained with more or less sophisticated discrimination techniques. Most commonly, single mode behavior is observed for operation near threshold, while for high levels of excitation, other modes begin to oscillate. The purpose of this study is to investigate the range of operating conditions under which single mode operation might be expected to occur spontaneously. It is shown that the ratio of homogeneous to inhomogeneous linewidths can be inferred from the multimode threshold.

There are several basic effects that can give rise to multimode oscillation in lasers. For high gain lasers operating very close to threshold, it is common to observe a distribution of cavity modes having a spectral envelope that narrows with increased pumping. ${ }^{1}$ This effect is simply a manifestation of the intense spontaneous emission that is feeding the various cavity modes and the relatively weak selective enhancement of the mode closest to line center. If the laser has a substantial homogeneous line-broadening component, such multimode oscillations always cease when the pumping rate has been raised to a few percent above threshold.

For higher values of the pumping rate, it sometimes happens that the laser reverts to multimode operation. This effect can provide a simple measure of the relative amounts of homogeneous and inhomogeneous broadening. In a laser with substantial inhomogeneous broadening, it is possible for the gain curve to reach the oscillation threshold over a range of frequencies, and the value of the threshold parameter at which additional modes first appear is a direct indication of the broadening ratio. While it is well known that inhomogeneously broadened lasers can oscillate in many cavity modes, ${ }^{2.3}$ this important transition from single mode to multimode behavior has not previously been examined in detail.

In the following analysis, it is assumed that there is a single strongly saturating longitudinal mode near line center in a laser oscillator. If the gain in the vicinity of line center is an increasing function of the frequency difference from line center, it is concluded that additional cavity modes would be able to oscillate. If the gain decreases away from line center, only one mode can oscillate. The equations needed to carry out this calculation can be developed in various ways. It is quickest, though, to simply quote them from a somewhat related analysis of a pulsation instability of laser oscillators. ${ }^{4}$ Thus, the saturating intensity $s I$ of a line center mode in a standing-wave gas laser is related to the threshold parameter $r$ by the equation

$\frac{1}{r}=\int_{-\infty}^{\infty} \frac{N(v) d v}{1+(k v / \gamma)^{2}+2 s I} / \int_{-\infty}^{\infty} \frac{N(v) d v}{1+(k v / \gamma)^{2}}$,

where $N(v)$ is the longitudinal velocity distribution of the population inversion, $k$ is the propagation constant, and $\gamma$ is the phase relaxation rate. This rate is related to the homogeneous linewidth by $\gamma=\pi \Delta v_{h}$. The threshold parameter $r^{\prime}$ needed for oscillation of a neighboring mode that is separated from line center by the radian frequency $\Delta \omega$ can be written

$$
\begin{aligned}
\frac{1}{r^{\prime}}= & \int_{-\infty}^{\infty} \frac{1+(k v / \gamma)^{2}}{1+[(\Delta \omega-k v) / \gamma]^{2}} \frac{N(v) d v}{1+(k v / \gamma)^{2}+2 s I} / \\
& \int_{-\infty}^{\infty} \frac{N(v) d v}{1+(k v / \gamma)^{2}} .
\end{aligned}
$$

If $r^{\prime}$ is less than $r$, multimode oscillation will be observed. The corresponding equations for a ring laser may be



FIG. 1. Normalized intensity $s I$ vs the threshold parameter $r$ for various values of the mixed broadening parameter $\rho=\epsilon^{2}=\Delta v_{h}{ }^{2} \ln 2 / \Delta v_{i}^{2}$. For small values of $\rho$, the curve approaches the relationship $s I=\left(r^{2}-1\right) / 2$, and for large $\rho$, the curve approaches $s I=(r-1) / 2$. 


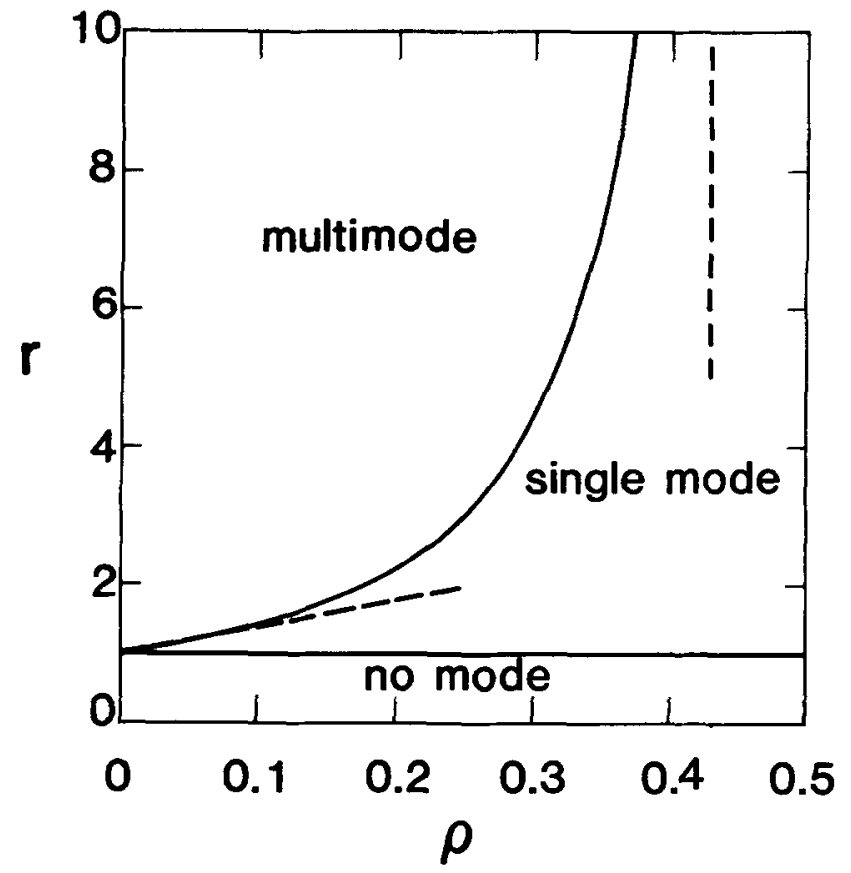

FIG. 2. Threshold curves for single mode and multimode oscillation as a function of the mixed broadening parameter $\rho$. The dashed lines mark the asymptotic limits described in the text, and below $r=1$, no lasing is possible.

shown to be of the same form if $2 s I$, the two way intensity, is replaced by $s I$. The equations are the same again for a travelling wave non-Doppler inhomogeneously broadened laser, but they become somewhat more complicated for a standing-wave non-Doppler laser ${ }^{5}$ if spatial hole burning is significant. ${ }^{6}$

As a first step in the analysis, it is convenient to introduce the normalized frequency difference $V=k v / \gamma$ and to assume that the velocity distribution for more generally the inhomogeneous line shape function) is Gaussian. Thus, Eqs. (1) and (2) reduce to

$$
\begin{aligned}
& \frac{1}{r}=\int_{-\infty}^{\infty} \frac{\exp \left(-\epsilon^{2} V^{2}\right) d V}{1+V^{2}+2 s I} / \int_{-\infty}^{\infty} \frac{\exp \left(-\epsilon^{2} V^{2}\right) d V}{1+V^{2}} \\
& \frac{1}{r^{\prime}}=\int_{-\infty}^{\infty} \frac{1+V^{2}}{1+(U-V)^{2}} \frac{\exp \left(-\epsilon^{2} V^{2}\right) d V}{1+V^{2}+2 s I} \\
& / \int_{-\infty}^{\infty} \frac{\exp \left(-\epsilon^{2} V^{2}\right) d V}{1+V^{2}}
\end{aligned}
$$

where the frequency displacement $U=\Delta \omega / \gamma$ and the natural damping ratio $\epsilon=\Delta v_{h}(\ln 2)^{1 / 2} / \Delta v_{i}$ have been introduced with $\Delta v_{i}$ the width of the inhomogeneous frequency distribution.

The multimode oscillation condition mentioned previously can now be evaluated by differentiation of Eq. (4) with respect to $U$ to determine whether or not the gain increases away from line center. In particular, the threshold for multimode oscillation can be determined by obtaining the second derivative of $\left(1 / r^{\prime}\right)$, evaluating it at $U=0$ and setting the result equal to zero. This condition is found to be

$$
0=\int_{-\infty}^{\infty} \frac{\left(1-3 V^{2}\right) \exp \left(-\epsilon^{2} V^{2}\right) d V}{\left(1+V^{2}\right)^{2}\left(1+V^{2}+2 s I\right)}
$$

The solutions of Eq. (3) are plotted in Fig. 1 for various values of the line broadening parameter $\rho=\epsilon^{2}$ and these plots enable one to predict the output of a single mode laser with mixed line broadening. A simultaneous solution of Eqs. (3) and (5) is given in Fig. 2, and this is the principal result of the present study. For any given line-broadening parameters and threshold parameter, one can tell immediately whether or not a given laser will operate in more than one mode. Alternatively, if one determines the value of the threshold parameter at which a laser switches to multimode operation, then the ratio of homogeneous to inhomogeneous linewidth can be easily found.

It should be noted that this second derivative calculation of the multimode threshold is only rigorously valid if the cavity mode spacing is small compared to the saturationbroadened homogeneous linewidth (the characteristic width of spectral holes). If this condition is not satisfied, the previous result [Eq. (4)] may still be used to test whether a second mode at frequency displacement $U$ is above threshold. It may be also be observed from these calculations that for $\rho>0.4282$, multimode oscillation is impossible for any value of $r$, and this maximum value of $\rho$ is marked on the graph. ${ }^{7}$ Various analytical approximations can also be obtained and the lowest order limit $r=1+4 \rho$ is shown in the figure.

To illustrate these results, we include a few numerical examples. For the broadening parameters $\Delta v_{h}=\Delta v_{i}$, one finds $\rho=0.69$, and no other modes are possible. With $\Delta v_{h}$ $=0.5 \Delta v_{i}$, one finds $\rho=0.17$, and from Fig. 2, other modes commence at $r \simeq 2$. With $\Delta v_{h}=0.1 \Delta v_{i}$ one finds $\rho=0.0069$, and multimode operation begins almost at the lasing threshold $r=1$. Alternatively, one might determine in the laboratory that a particular laser begins multimode oscillation at $r=3$, for example. It follows from the graph that $\rho \simeq 0.25$ and hence $\Delta v_{h}=0.6 \Delta v_{i}$.

In conclusion, we mention as a specific practical example the well-known multimode behavior of GaAs lasers. Carrier diffusion in GaAs is fast enough that longitudinal spatial-holeburning effects should be unimportant, and hence, if the amplifying medium were mainly homogeneously broadened, single mode oscillation should predominate. ${ }^{6}$ Indeed, single mode oscillation is observed under a wide variety of conditions. However, as GaAs lasers age, there are indications that the effective inhomogeneous linewidth begins to increase. Thus, there is a broadening with age of the overall spectral output. ${ }^{8}$ Also, high-frequency spontaneous pulsations begin to occur, ${ }^{9}$ and the semiclassical laser models show that the threshold for such pulsations can be very low $(r \simeq 1)$ in inhomogeneously broadened lasers-in contrast to homogeneous systems where some other pulsation mechanism has to be postulated. ${ }^{5}$ Thus, a detailed study of the multimode oscillation characteristics of GaAs might provide valuable data on the effective linewidths and new insight into the spontaneous pulsation phenomena. ${ }^{10}$

${ }^{1}$ L. W. Casperson, J. Appl, Phys. 46, 5194 (1975).

${ }^{2}$ A. E. Siegman, An Introduction to Lasers and Masers (McGraw-Hill, New York, 1971), Sec. 9.4 
${ }^{3}$ A. Yariv, Introduction to Optical Electronics, 2nd ed. (Holt, Rinehart and Winston, New York, 1976), Sec. 6.6.

${ }^{4}$ L. W. Casperson, Phys. Rev. A 21, 911 (1980). Equations (70) and (71) correspond to Eqs. (1) and (2) in the present study.

${ }^{5} \mathrm{~L}$. W. Casperson, Phys. Rev. A 23, 248 (1981).

${ }^{6}$ H. G. Danielmeyer, J. Appl. Phys. 42, 3125 (1971).

'In lasers with spatial hole burning multimode oscillation may become possible at large values of $r$. See Ref. 6.

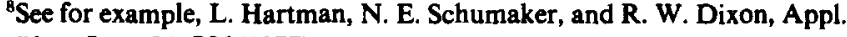
Phys. Lett. 31, 756 (1977)

'T. L. Paoli, IEEE J. Quantum Electron. QE-13, 351 (1977).

${ }^{10}$ The relationship between multimode oscillation and spontaneous pulsations has often been noted. See for example, H. Bachert, P. G. Eliseev, M. A. Manko, V. P. Strahov, and C. M. Thay, IEEE J. Quantum Electron. QE-11, 507 (1975). 